Didáctica. Lengua y literatura

ISSN-e: 1988-2548

\title{
Actitudes y hábitos lingüísticos entre el alumnado del Grado en Educación Infantil (UIB) ${ }^{1}$
}

\author{
Rosa Calafat Vila²; Àlvaro Calero-Pons ${ }^{3}$
}

Fecha revisión: 31-01-2019 / Fecha aceptación: 21-05-2019

Resumen. En la adquisición de una lengua, la competencia pragmática resulta imprescindible, puesto que de su conocimiento se deben derivar hábitos y actitudes positivas hacia el entorno. Este hecho tiene una especial relevancia cuando se trata del aprendizaje de una lengua minorizada, obligada a compartir funciones con otra de mayor peso simbólico. En este artículo, analizamos las percepciones lingüísticas de uno de los tres grupos de primer año que han cursado, a lo largo del primer cuatrimestre del año académico 2017-2018, la asignatura Lengua Catalana y su Didáctica, del Grado en Educación Infantil de la Universidad de las Illes Balears (UIB). Pretendemos confirmar la correlación entre actitudes y hábitos favorables al catalán con el logro de los objetivos curriculares de aprendizaje. La influencia del entorno, más allá de las políticas lingüísticas que se aplican para la normalización del catalán en la docencia universitaria balear, acaba determinando el rendimiento lingüístico del alumnado.

Palabras clave: actitudes lingüísticas; hábitos lingüísticos; sistema educativo; catalán como L1 y L2 en las Islas Baleares; política lingüística.

\section{[en] Language attitudes and habits among students of the Bachelor's Degree in Early Childhood Education (UIB)}

\footnotetext{
Abstract. When acquiring a language, pragmatic competence becomes essential, given that positive attitudes and habits towards the environment have to be derived from its knowledge. This fact assumes a particular relevance when it comes to learning a minoritized language, which is forced to share functions with other language having more symbolic weight. In this paper, we analyze the linguistic perceptions of one of three first year groups which have taken the subject Catalan Language and its Teaching, from the Bachelor's Degree in Early Childhood Education at University of the Balearic Islands (UIB), in the first four months of the school year 2017-2018. We intend to confirm the correlation between favorable attitudes and habits towards Catalan with the curricular learning objectives. Beyond the linguistic policies which are applied to the normalization of Catalan in the Balearic university teaching, the influence of environment determines the students' linguistic performance.

Keywords: linguistic attitudes; linguistic habits; educational system; Catalan as L1 \& L2 in the Balearic Islands; linguistic policy.

1 Este artículo ha sido elaborado gracias a la financiación del proyecto FFI 2016-80191-P "Extensión social de la norma lingüística en los medios de comunicación durante el siglo xx" (2016-2020), concedido por el Ministerio de Educación, Cultura y Deporte; así como al Grup de Recerca en Història de la Llengua Catalana de l'Època Contemporània, de la Universitat Autònoma de Barcelona (UAB), y a un contrato predoctoral concedido por la Universidad de las Illes Balears al doctorando.

2 Departamento de Filologia Catalana i Lingüística General, Universitat de les Illes Balears rosa.calafat@uib.cat

3 Departamento de Filologia Catalana i Lingüística General, Universitat de les Illes Balears alvaro.calero@uib.cat
} 


\section{[fr] Attitudes et habitudes linguistiques des étudiants de la Licence en éducation pré-primaire (UIB)}

Resumé. Dans l'acquisition d'une langue, la compétence pragmatique devient indispensable, compte tenu que de sa métrise doivent se découler des habitudes et des attitudes positives vers l'environnement. Cela est particulièrement important lorsqu'il s'agit de l'apprentissage d'une langue minorisée, forcée à partager des fonctions avec une autre langue ayant un poids symbolique plus grand. Dans cet article, nous analysons les perceptions linguistiques d'un des trois groupes de la première année qui ont suivi, tout au long du premier quadrimestre de l'année universitaire 2017-2018, la matière Langue catalane et sa didactique, dans la Licence en éducation pré-primaire de l'Université des Îles Baléares (UIB). Nous nous proposons de vérifier la corrélation entre les attitudes et les habitudes favorables au catalan avec la réalisation des objectifs curriculaires pédagogiques. L'influence de l'environnement, au delà des politiques linguistiques mises en œuvre pour la normalisation du catalan dans l'enseignent universitaire baléare, déterminent les performances linguistiques des élèves.

Mots-clés: attitudes linguistiques; habitudes linguistiques; système éducatif; catalan comme L1 et L2 aux Îles Baléares; politique linguistique.

Sumario. 1. Introducción. 2. Competencias lingüísticas y educación en las Islas Baleares. 3. Metodología. 3.1. Perfil del encuestado. 4. Actitudes lingüísticas. 4.1. Actitudes del Grupo encuestado, según lugar de residencia. 4.1.1. El catalán, lengua de rotulación. 4.1.2. El catalán, en el ámbito público y privado. 4.1.3. El catalán, en la docencia. 4.1.4. El catalán, idioma de cohesión social y de mercado. 5. Hábitos lingüísticos. 5.1. Hábitos del Grupo encuestado, según lugar de residencia. 6. Conclusiones. Bibliografía. Anexo.

Cómo citar: Calafat Vila, R.; Calero-Pons, Á. (2019). Actitudes y hábitos lingüísticos entre el alumnado del Grado en Educación Infantil (UIB), Didáctica. Lengua y literatura, 31, 243-261.

\section{Introducción}

La lengua catalana está presente en cuatro estados europeos, España, Francia, Andorra e Italia. Además, dentro de España, la comunidad lingüística catalana se encuentra dividida por comunidades autónomas: Cataluña, Valencia, las Islas Baleares, Aragón y Murcia. Su contexto sociolingüístico varía según las políticas lingüísticas que se aplican (Argenter, 2014, 7-9). Si atendemos al sistema educativo, mientras que en Cataluña y Baleares este idioma tiene reconocimiento vehicular, en la comunidad valenciana existe la elección de lengua, lo que pudiera explicar el menor conocimiento que esta población tiene de su lengua autóctona ${ }^{4}$.

Las leyes autonómicas, en materia educativa, entran en tensión con las ministeriales desde el momento en que se pretende dar al catalán un funcionamiento autónomo (Pradilla Cardona, 2017, 234 y ss.; Bastardas i Boada, 2002, 8). El Estatuto de Autonomía de las Islas Baleares y el de Cataluña garantizan la oficialidad del español y del catalán, aunque solo reconocen como idioma propio a este último. Las directrices de política lingüística, que emanan de las leyes de estas autonomías, dan valor referencial al idioma catalán convirtiéndolo en el símbolo de cohesión de su comunidad lingüística. El artículo 5 del Estatuto balear y el 12 del catalán, así lo establecen: "El Govern ha de promoure la comunicació, l'intercanvi cultural i la cooperació amb les comunitats i els territoris, pertanyents o no a l'Estat espanyol, que tenen vincles 
lingüístics i culturals amb les Illes Balears". En cambio, las leyes de la comunidad valenciana, en materia lingüística, se ciñen a sus límites autonómicos: "tot allò que és valencià en un concepte cultural propi en l'estricte marc geogràfic que abasta"s.

La comunidad lingüística catalana comparte funciones con el castellano, lengua de Estado. El panorama europeo nos procura otros casos análogos, como el del neerlandés, que debe compartir espacio lingüístico con el francés en la parte suroeste de Flandes (Cotano, 2000, 74). Europa vive dinámicas de tensión y cambio lingüístico (Poggeschi, 2004, 1), en que las lenguas minorizadas, con menos recursos legales y simbólicos, deben esforzarse por sobrevivir (Moreno Cabrera, 2009, 163). Los hablantes de estas lenguas condicionadas pueden adoptar actitudes desfavorables en torno a su propia lengua y cultura. De ahí que el Consejo de Europa, recientemente, haya incidido en la expresión cultural del idioma, por encima de los conocimientos lingüísticos, con el fin de trabajar nuevas motivaciones y nuevos hábitos que permitan revertir situaciones de subordinación lingüística (Council of Europe, 2007a, 114-115).

En el ámbito catalán, se han desarrollado planes de política lingüística ${ }^{6}$ para fomentar la lealtad de sus hablantes. Si bien, según estudios realizados, las actitudes lingüísticas del alumnado son independientes de su origen, los que provienen de países que han aplicado políticas monolingüistas muestran una tendencia a presentar actitudes negativas hacia el estudio del catalán (Estors Sastre, 2014: 169-170; Bretxa, Comajoan y Sorolla, 2009, 13-14).

Los recientes hitos en la planificación de este idioma se asientan, fundamentalmente, en el sistema educativo. Sirva de ejemplo la evolución ascendente en el uso del catalán en los exámenes de selectividad de las Islas Baleares: En el 2015, el 81.1 \% de los alumnos se decantaron por el idioma autonómico 7 . Aunque el Gobierno Bauzá del Partido Popular, 2011-2015, adoptó medidas para revertir la política lingüística educativa hasta el momento en vigor (Ballester Cardell, 2016, 89 y ss.), esta se mantuvo firme ${ }^{8}$. Se demostró la eficacia del movimiento llamado escola catalana, iniciado en torno a los años ochenta del siglo pasado?

Si bien la educación es uno de los pilares para la (re)construcción y normalización de un idioma, no es en sí suficiente (Bourdieu y Passeron, 1977). En el caso catalán, la pretensión institucional es hacer de este idioma lengua vehicular y de acogida para los nuevos hablantes ${ }^{10}$. Esta aspiración topa con las leyes

5 Gifreu (2011 y 2014) sostiene que el proceso de normalización del catalán no se consolidará a menos que su espacio de comunicación, entendido como una red de medios audiovisuales y escritos, esté políticamente institucionalizado y socialmente reconocido, en cuanto a las consultas de los textos legales mencionados.

6 El portal EINESCAT enlaza la web Històries del català, en la que se recopilan, en formato vídeo, los testimonios de personas, repartidas por toda la geografía de las islas, que cuentan su experiencia sobre cuándo decidieron aprender catalán y utilizarlo en su día a día. (Consell de Mallorca. 2018: EINESCAT. Disponible en http://www. conselldemallorca.net/?id_section=8277 [Consulta: 26 de noviembre de 2018].)

La Guia pràctica d'acollida lingüística muestra que uno de los incentivos que encuentran para hablarlo es que no solo expresan que están aquí, sino que también son de aquí. (Plataforma per la llengua. 2009: Guia pràctica d'acollida lingüística, Palma, Consell de Mallorca, 1 y 3. Disponible en http://www.conselldemallorca.net/ media/15943/guia.pdf [Consulta: 26 de noviembre de 2018].)

7 Véase los gráficos del Grup de Recerca Sociolingüística de les Illes Balears (GRESIB). Disponible en http:// www.uib.cat/depart/dfc/gresib/selectivitat.htm [Consulta: 26 de noviembre de 2018].

8 A la consulta de qué lengua escogían los progenitores para escolarizar a sus hijos, un $83 \%$ se decidieron por el catalán (Bibiloni, 2015, 131).

9 Véase: http://www.somescola.cat/

10 Véase el portal institucional del Gobierno catalán en cuanto a la simbolización de la lengua. Disponible en http://web.gencat.cat/ca/temes/llengua/ [Consulta: 26 de noviembre de 2018]. 
estatales ${ }^{11}$, cuyo peso referencial ha conducido al bilingüismo asimétrico (Bibiloni, 2014, 91), actualmente naturalizado entre hablantes de catalán. Así, las instancias autonómicas se han visto obligadas a promover políticas para el cambio de las actitudes en los autóctonos, sometidos a la presión referencial del castellano, y a desarrollar planes de inmersión de la población migrada ${ }^{12}$.

En el contexto sociolingüístico descrito, hemos elaborado la Enquesta d'actituds i habilitats lingüístiques, Grau d'Educació Infantil (2017-2018) (anexa al final del artículo), para medir las percepciones hacia el catalán de uno de los tres grupos de primer año de la asignatura Lengua Catalana y su Didáctica, del Grado en Educación Infantil de la Universidad de las Illes Balears. Este alumnado de nuevo ingreso proviene mayoritariamente de centros públicos en que se han aplicado políticas lingüísticas para el fomento del catalán.

\section{Competencias lingüísticas y educación en las Islas Baleares}

Desde 1997 el sistema educativo balear se rige por el Decreto de Mínimos ${ }^{13}$, cuyo texto establece que al menos el $50 \%$ de la escolarización se debe impartir en catalán, sin especificar su máximo; lo cual ha permitido desarrollar políticas lingüísticas extensivas.

Durante el Gobierno Bauzá, se intentó, mediante la introducción del inglés, alterar la presencia de la lengua propia, lo que provocó una gran oposición académica y social (Parcerisa y Verger Planells, 2016, 358 y 360). La aprobación del Decreto 15/2013 (Govern de les Illes Balears, 2013) del tratamiento integral de lenguas (TIL), recibió el voto negativo de todos los partidos de la oposición y generó un fuerte movimiento de oposición social, conocido como la "Marea Verda".

En 2015, con el cambio de gobierno, se restituyó el Decreto de Mínimos. Así mismo, no debe ignorarse la existencia de centros, aunque minoritarios, que no lo aplican (Munar i Munar, 2009, 93 y 95).

En la enseñanza superior, la Universidad de las Illes Balears (UIB) garantiza la presencia mayoritaria del catalán. El artículo 4.1 de sus estatutos establece que se fomentará y normalizará el uso del catalán en el ámbito de sus competencias ${ }^{14}$. Así pues, en el curso 2015-2016, de las 179 asignaturas que se impartieron en el Grado de Educación Infantil $1^{15}$, el $88.3 \%$ se hicieron en catalán; el $7.3 \%$, en castellano; y el $4.5 \%$, en inglés. Este porcentaje, a favor de la lengua propia, acredita, que no certifica, el nivel C1 de catalán para los graduados; con el requisito, por orden ministerial ${ }^{16}$ de cursar la asignatura Aprendizaje de las Lenguas en Contextos Multilingües.

11 Véase el dossier Un estat contra l'escola en català, publicado en la revista Presència, el 11 de enero de 2015. Consta en el repositoro de L'Institut d'Estudis Catalans (IEC): http://blogs.iec.cat/she/wp-content/uploads/sites/30/2015/03/dossier-catal \%C3 \%A0-a-lescola.pdf

12 Véase la Direcció General de Política Lingüística (Govern de les Illes Balears). Disponible en http://www.caib. cat/govern/organigrama/area.do?lang=ca\&coduo=2390443 [Consulta: 26 de noviembre de 2018].

13 Decreto 92/1997 (Govern de les Illes Balears, 1997). En cuanto a su creación y desarrollo, véase la entrada correspondiente en la Enciclopèdia catalana: http://enciclopedia.cat/EC-GEC-0522727.xml

14 Véase: https://seu.uib.cat/fou/acord/94/9439.html\#4

15 Datos proporcionados por el Servei d'Estadística i Qualitat Universitària (SEQUA) de la Universitat de les Illes Balears.

16 Véase la Orden ECI/3857/2007: https://www.boe.es/diario_boe/txt.php?id=BOE-A-2007-22449 
Ahora bien, el hecho de que el catalán goce de políticas para su fomento en el ámbito educativo no resuelve las actitudes de sus hablantes, sometidos estos a la presión del español (Bastardas i Boada, 2012, 79 y 83 y ss.). Así parecen constatarlo los resultados de la encuesta sobre el conocimiento lingüístico del alumnado de nuevo ingreso en el Grado en Educación Infantil de la universidad balear. Fueron encuestados en 2015, en el momento de la matrícula, con una participación del 96.9 $\%$ (126 respuestas de 130). Estos alumnos debían seleccionar la opción que consideraban que se ajustaba más a su situación. Cada una representaba uno de los niveles lingüísticos que determina el MECR (A1, A2, B1, B2, C1 y C2). Según datos del 2015-2016 proporcionados por el Servei d'Estadística i Qualitat Universitària (SEQUA) de la Universidad Balear, estos alumnos, procedentes de centros docentes en que el catalán puede haber tenido una presencia mayoritaria, reconocen poseer un grado de conocimiento y confianza ligeramente superior en español. A tenor de ello, podríamos sugerir que para el cambio de las actitudes lingüísticas de los hablantes se requiere reflexionar sobre ellas a partir del aprendizaje pragmático o cultural (Bastardas i Boada, 2002, 92; Joan i Marí, 2008, 35; Iranzo García y Queralt i Catà, 2010, 66; Navarro, Huguet y Sansó, 2016, 239-240).

\section{Metodología}

Siguiendo las recomendaciones del manual de elaboración de encuestas de Cea d'Ancona (2004), elaboramos un cuestionario anónimo que nos permitiera valorar las actitudes y percepciones hacia la lengua catalana, Enquesta d'actituds i habilitats lingüístiques, Grau d'Educació Infantil (2017-2018) (en adelante, Enquesta d'actituds). A partir de 73 ítems, medidos según la escala de Likert, tomamos como muestra a los 40 alumnos de la asignatura Lengua Catalana y su Didáctica, del primer curso del Grado en Educación Infantil, 2017-2018. Además de la asignatura específica de lengua catalana, este alumnado cursa otras ocho asignaturas, de las cuales siete son en catalán y una en español, La Lengua en el Aula de Infantil: del Placer de la Lectura al Juego de la Escritura, a tenor de lo que reflejan las guías docentes $^{17}$. En cuanto a las seis asignaturas que se ofrecen como optativas, dos figuran en catalán, dos en español y otras dos en inglés. Mientras que la específica de lengua catalana, de donde procede la muestra encuestada, se imparte en el primer curso, su homóloga en español se cursa en el último año, Lengua Española: Estrategias para la Comunicación.

En el estudio, se logró una participación del $82.5 \%$, correspondiendo al 33 de los 40 sujetos del grupo. El cuestionario se realizó durante la primera semana lectiva, al final de la clase de la correspondiente asignatura.

Los sujetos encuestados responden a tres grupos, según su relación con el catalán: a) los que tienen este idioma como lengua primera (L1), que declaran hablarlo normalmente o siempre; y tener competencias lingüísticas buenas o muy buenas; b) los hablantes de catalán como segunda lengua (L2), que lo hablan en casa raramente o nunca, aunque afirman tener buenas o muy buenas competencias; y, c) los que poseen el catalán como una tercera lengua (L3), que raramente lo hablan

17 Véase el plan de estudios: http://estudis.uib.cat/grau/infantil/GEI2-P/assignatures.html 
y reconocen que sus competencias son malas o muy malas ${ }^{18}$. Se establece, así, una jerarquía entre los hablantes. En este sentido, el Consejo de Europa explica que una L2 puede tener una presencia muy reducida, casi nula, en algunos ámbitos, hasta llegar a percibirse como una simple materia en los centros educativos (Council of Europe, 2007a, 52 y 114 y ss.). Entre los encuestados, los alumnos del grupo L3, tienden a priorizar la certificación sin contemplar su incorporación en la comunidad catalanohablante.

Al amparo del Consejo de Europa, la primera lengua (L1) es la que se adquiere desde la infancia, antes del periodo crítico (2-3 años); puede haber más de una y se prefiere este término al de lengua materna, más popular, puesto que, generalmente, no es solo la lengua de la madre, sino del entorno (lengua familiar). La segunda lengua (L2) es la lengua propia de un territorio, donde está presente y tiene presencia social -se usa para la comunicación-pero no se puede considerar L1 para un individuo. Las necesidades en la enseñanza de una L2 son diferentes de las de una lengua extranjera (LE), ya que esta no es oficial en ese territorio, sino en otro estado, y no se suele tener oportunidad de hablarla más allá de la enseñanza reglada, mientras que la L2 forma parte de la vida diaria: “A ‘foreign' language is typically one spoken widely and often as official/national language in another country with which the learner has limited or no contact except in the classroom, whereas a 'second' language surrounds the learner on a daily basis" (Council of Europe, 2007b, s. n.).

Porcentualmente, el $36.36 \%$ de la muestra forma parte del grupo L1; de los cuales, el $50 \%$ reside en Palma, a partir de ahora se usará indistintamente el término ciudad o Palma; y el $50 \%$ en el resto de Mallorca. Un 39.39 \% son L2; de estos, el $81.25 \%$ vive en Palma y el $18.75 \%$, en el resto de Mallorca. Por último, el $24.24 \%$ tiene el catalán como L3, y todos residen en la ciudad. La Enquesta d'actitud revela que el $63.63 \%$ de la muestra no tiene el catalán como L1. De estos, el $4.8 \%$ han nacido en otra comunidad autónoma - de habla no catalana-y el $4.8 \%$, en otro país de habla hispana; lo cual nos proporciona un $90.4 \%$ de L2 nacidos en Mallorca. A su vez, la muestra permite constatar la concentración de castellanoparlantes en la ciudad. Se confirman así los datos que detalla la Enquesta d'Usos Lingüístics a les Illes Balears $2014^{19}$, en cuanto a la ventaja de hablantes de castellano en Baleares.

\subsection{Perfil del encuestado}

Atendiendo a las tesis de Casas-Deseures y Comajoan Colomé $(2015,192)$, en cuanto a la capacidad lingüística de los alumnos y su relación con la vía de ingreso en la universidad, constatamos que, entre los sujetos de la Enquesta d'actituds, la opción del Bachillerato solo es mayoritaria entre los que tienen el catalán como L1 y provienen de Palma (tabla 1), según se deriva de la pregunta número 3, "Vía de acceso a la Universidad", formulada en la citada encuesta.

\footnotetext{
18 Los alumnos con capacidades regulares para la lectura y escritura se han mantenido en el grupo L2. Los que han indicado que tienen como regulares la expresión y compresión oral se han integrado en los L3.

19 Disponible en http://blogs.iec.cat/cruscat/2017/11/07/enquesta-dusos-linguistics-a-les-illes-balears-2014-analisi/ [Consulta: 26 de noviembre de 2018].
} 
Tabla 1. Vía de acceso a la Universidad del grupo encuestado

\begin{tabular}{|l|c|c|c|c|}
\cline { 2 - 5 } \multicolumn{1}{c|}{} & Bachillerato & Ciclo formativo & Mayores de 25 años & Otros \\
\hline L1 (ciudad) & $67 \%$ & $33 \%$ & $0.0 \%$ & $0.0 \%$ \\
\hline L1 (resto de Mallorca) & $33 \%$ & $67 \%$ & $0.0 \%$ & $0.0 \%$ \\
\hline L2 (ciudad) & $31 \%$ & $69 \%$ & $0.0 \%$ & $0.0 \%$ \\
\hline L2 (resto de Mallorca) & $33 \%$ & $67 \%$ & $0.0 \%$ & $0.0 \%$ \\
\hline L3 (ciudad) & $37.5 \%$ & $50 \%$ & $12.5 \%$ & $0.0 \%$ \\
\hline
\end{tabular}

El estudio que realizamos se basa en la comparación entre los grupos L1, L2 y L3, sometidos a la variable de residencia; según sean de Palma o del resto de Mallorca. Atendiendo al mayor grado de castellanización que presenta la ciudad (Marimon Riutort, 2015, 97 y ss.; Melià, 2015, 63 y 66 y ss.), esta distribución puede afectar a las actitudes y a los hábitos de los sujetos analizados.

Este estudio da la consideración de ciudad al conjunto que incluye el municipio de Palma, propiamente, y los de su radio de influencia más fuerte: Calvià, Marratxí y Llucmajor.

Tabla 2. Alumnos de primera matrícula, según residencia

\begin{tabular}{|l|c|c|}
\hline \multicolumn{1}{|c|}{ ¿Es la 1. ${ }^{\text {a }}$ matrícula? } & Sí & No \\
\hline L1 (ciudad) & $17 \%$ & $83 \%$ \\
\hline L1 (resto de Mallorca) & $50 \%$ & $50 \%$ \\
\hline L2 (ciudad) & $23 \%$ & $77 \%$ \\
\hline L2 (resto de Mallorca) & $33 \%$ & $67 \%$ \\
\hline L3 (ciudad) & $25 \%$ & $75 \%$ \\
\hline
\end{tabular}

\section{Actitudes lingüísticas}

Los setenta y tres ítems del cuestionario, analizados globalmente, nos permiten medir, por una parte, las actitudes de los sujetos encuestados, y por otra sus hábitos lingüísticos.

En cuanto a las actitudes, se ha pretendido medir la disposición a la presencia del catalán en los espacios urbanos - rotulación-, en los administrativos y en la docencia. Se han clasificado como "positivas", para el uso social del catalán, las respuestas "de acuerdo" y "totalmente de acuerdo" a las preguntas sobre la presencia mediática de esta lengua (de la 63 a la 73). Se han considerado negativas las respuestas "en desacuerdo" y "totalmente en desacuerdo"; y neutras, "ni de acuerdo ni en desacuerdo", opción asimilable al "no sabe, no contesta". Se observa como las actitudes positivas hacia el uso del catalán aumentan a mayor contacto social con esta lengua, siendo más favorables entre los habitantes del resto de Mallorca que en los de la ciudad (figuras 1, 2, 3, 4 y 5). 
4.1. Actitudes del Grupo encuestado, según lugar de residencia
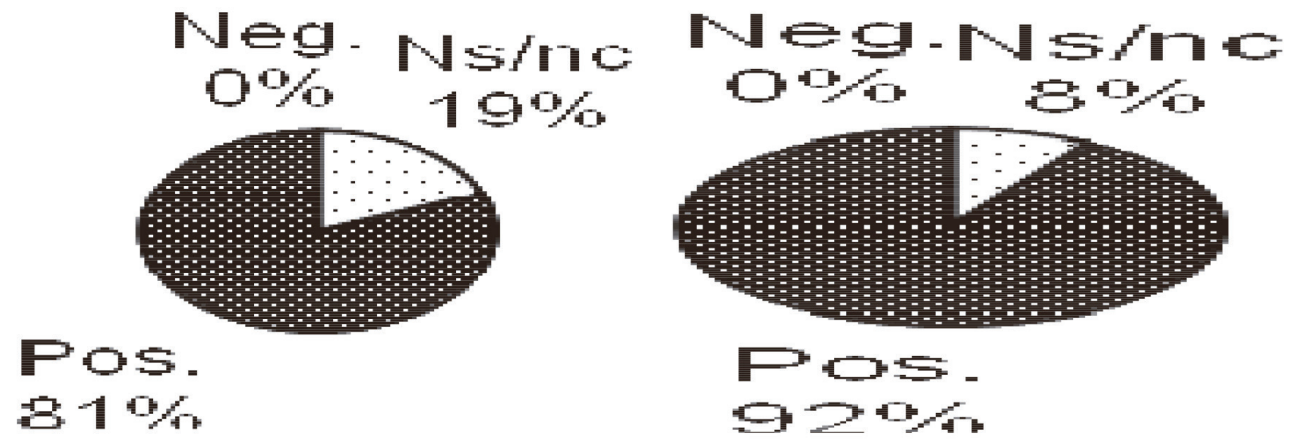

Figura 1. L1 (ciudad)

Figura 2. L1 (resto de Mallorca)

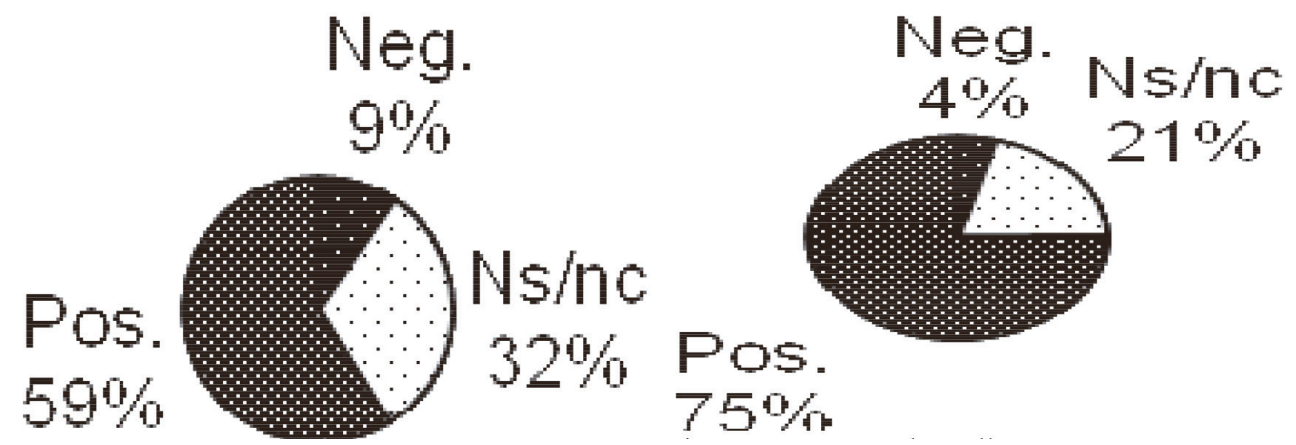

Figura 3. L2 (ciudad)

Figura 4. L2 (resto de Mallorca)

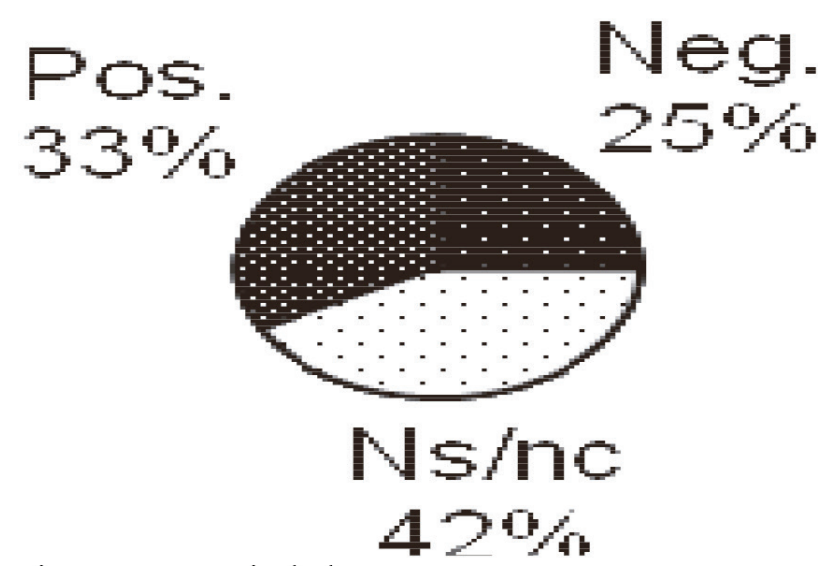

Figura 5. L3 (ciudad) 


\subsubsection{El catalán, lengua de rotulación}

Entre los encuestados solo un $67 \%$ de los palmesanos L1 consideraron que todo debería estar rotulado, como mínimo, en catalán, sin perjuicio de que lo estuviera también en otras lenguas, tal como se refleja en la pregunta 72 . Este porcentaje aumenta al $100 \%$ en los del resto de Mallorca. La aceptación en los L2 baja hasta el $54 \%$ para la ciudad y hasta el $33 \%$ para el resto de Mallorca; y es de un $25 \%$ entre los L3. Las actitudes contrarias aparecen en el grupo L2 en un $16 \%$ para la ciudad y en un $33 \%$ para el resto de la isla, así como en un $25 \%$ de los L3.

\subsubsection{El catalán, en el ámbito público y privado}

En general, todos los encuestados mantienen una predisposición positiva a la exigencia de las dos lenguas oficiales (ítems del 67 al 70), tanto en el ámbito público como en el privado ${ }^{20}$, aunque se tolera más la exigencia del idioma local en el primero (Melià, 2001, 8-9; Bibiloni, 2015, 129).

Si bien es cierto que no se dan actitudes negativas entre los L1, el $17 \%$ de los de la ciudad no se posicionan ante la presencia del catalán en el ámbito público y privado. En el resto de la isla, el $33 \%$ se mantienen al margen de la exigencia de lengua. Entre estos últimos, la aceptación del catalán sería mayor en la esfera pública.

Del mismo modo, las actitudes tienden a ser positivas entre los L2, solo un $15 \%$ de ellos consideraría normal la exclusividad del castellano en el sector privado. Entre los palmesanos, los que no se posicionan constituyen solo el $15 \%$ para el catalán y el $8 \%$ para el español, sin diferenciar sectores. En el resto de Mallorca, las actitudes son positivas en su totalidad. Entre estos, el porcentaje que no se posiciona, el $33 \%$, aparece referido al ámbito privado, sin diferenciar ambas lenguas.

Entre los L3, un $12.5 \%$ muestra una actitud negativa hacia la presencia del catalán en el sector público, y un $37.5 \%$ no se posiciona. Este último porcentaje se reduce al $12.5 \%$ al referirse al uso del castellano. En cuanto al sector privado, no se dan actitudes negativas explícitas hacia la lengua local; si bien, son más los que no se posicionan sobre la exigencia del catalán, un $50 \%$, que sobre la del español, un $25 \%$ de ellos se manifiestan a favor del uso del castellano.

Si medimos los usos lingüísticos por ámbitos específicos -preguntas de la 13 a la 24-, los L1 de la ciudad usan el catalán en un $67 \%$, en el ámbito laboral con los desconocidos, en los comercios y en la Universidad; mientras que los que viven en el resto de Mallorca, tanto si son L1 como L2 lo hacen un $83 \%$. Cabe reseñar que los L2 de la ciudad tienden a utilizar el español de manera casi exclusiva entre el vecindario, el ámbito sanitario y los comercios. Entre este colectivo, la presencia del catalán solo despunta en el entorno universitario: el $69 \%$ de ellos se decanta por el catalán en las interacciones con profesores y el $62 \%$ lo hace ante la administración académica.

20 En un estudio similar, Ibáñez Ferreté $(2014,227)$ considera pertinente extraer conclusiones de los usos lingüísticos para detectar los discursos ideológicos entre el estudiantado, a pesar de la dificultad de relacionar ideologías y usos, ya que tendrán la capacidad de influir en la sociedad venidera. 


\subsubsection{El catalán, en la docencia}

Los individuos que tienen el catalán como L1 se muestran favorables a que sea la lengua vehicular de la docencia. La diferencia entre ellos radica en que la opción de respuesta más contundente que nos ofrece la escala de Likert, "totalmente de acuerdo", solo sería mayoritaria entre los que viven en el resto de Mallorca, con un $67 \%$. La influencia del bilingüismo asimétrico, que se da en la ciudad con mayor intensidad, explicaría esta desviación.

Entre los que tienen el catalán como L2, la presencia de esta en el ámbito docente sería un hecho normalizado, tanto entre los que residen en la ciudad como en el resto de Mallorca (Munar i Munar, 2009, 93 y 95), sin que se encuentren actitudes negativas; no obstante, hay que destacar que el $31 \%$ de los palmesanos y el $33 \%$ de los demás mallorquines no se posicionan. Para el grupo L3, tan solo un $12.5 \%$ considera normal que el catalán sea lengua vehicular en la docencia, frente al $25 \%$ que se muestran contrarios. Cabe resaltar que un $62.5 \%$ de este grupo optaron por marcar "ni de acuerdo ni en desacuerdo".

\subsubsection{El catalán, idioma de cohesión social y de mercado}

No se dan actitudes negativas entre los encuestados L1 en el ámbito social y en el de mercado. Tan solo cabría destacar el $33 \%$ de la ciudad que se muestran indiferentes ante la posibilidad de que un desconocido se les dirija en catalán. Las actitudes negativas se dan entre los L2 de ciudad en los ámbitos que implican contactos interpersonales y productos de mercado. Ante la posibilidad de que un desconocido se les dirija en catalán, el $15 \%$ de los L2 de ciudad manifiestan desagrado, frente al $69 \%$ que se muestran indiferentes; solamente un $16 \%$ lo considerarían normal. En cambio, los L2 que viven en el resto de Mallorca no expresan opiniones negativas al respecto; y un $33 \%$ de ellos se muestra indiferente. Es en el grupo L3 donde se registran mayor número de percepciones negativas. Aunque el $50 \%$ de ellos escojan la respuesta "ni de acuerdo ni en desacuerdo", un $37.5 \%$, se muestran contrarios a ser abordados en catalán.

A su vez, la elección lingüística por el inglés como lengua de comunicación entre los encuestados, perfil joven y universitario, al dirigirse a un desconocido percibido como turista, aumenta entre los catalanohablantes del resto de Mallorca. Las respuestas a la pregunta 14, sobre la lengua de uso al dirigirse a desconocidos percibidos como extranjeros demuestran que los L1 de ciudad escogen el inglés un $33 \%$ en la situación descrita; frente al $83 \%$ del resto de Mallorca. Entre el grupo L2, los de Palma usan el inglés un $38 \%$; los del resto de Mallorca, un $67 \%$.

Al comparar el uso del castellano y del inglés como lengua de comunicación entre los sujetos encuestados, se confirma que la elección por el español, al dirigirse a un desconocido, tendría más peso entre los L1 -un $50 \%$ de ellos-y L2 -un $62 \%$ - residentes en Palma, que entre los habitantes del resto de Mallorca: el 17 \% y $33 \%$, respectivamente, de ellos se inclinan por usar el español. El colectivo L3 usa el inglés en un $62.5 \%$.

A las preguntas relacionadas con el consumo cultural mediático (de la 63 a la 65), los encuestados reflejan actitudes negativas al uso del catalán, mayoritariamente entre los que residen en Palma y tienen el catalán como L2 o L3. Al ser preguntados por las películas en catalán, el $38 \%$ de los palmesanos de L2 y el $62.5 \%$ de los L3 
expresan su desagrado ante la presencia de este idioma. Estos porcentajes se reducen al $8 \%$, entre los palmesanos de L2, y al $37.5 \%$, entre los L3, cuando se trata del consumo musical.

A la pregunta "Me gusta escuchar el catalán alrededor mío, aunque a veces no lo entienda todo" (ítem 63), los sujetos se muestran más tolerantes con la lengua local, puesto que no implica una interacción directa con ella. Aparece un porcentaje de indecisos en los palmesanos L2, del $31 \%$, que aumenta al $37.5 \%$ entre los L3.

\section{Hábitos lingüísticos}

La frecuencia con la que los sujetos de la muestra consumen productos culturales -películas, series y música- en catalán, en español o en terceras lenguas, tanto en versión original como doblados o subtitulados, debía darnos los datos sobre sus tendencias lingüísticas en este ámbito. Las preguntas comprendidas entre la 52 y la 62 se encargaban de establecer el medio de tal consumo: televisión, cine u otros.

A diferencia de lo que reflejan los gráficos sobre las actitudes, se observa que los hábitos lingüísticos negativos superan a los positivos entre los L2; acentuándose como muy negativos entre los L3 (figuras 8,9 y 10). Así mismo, y siguiendo la tónica general, los L2 del resto de Mallorca son más favorables al catalán ${ }^{21}$.

En cuanto a los L1 (figuras 6 y 7), se muestran más proclives al consumo lingüístico en su lengua propia. Los gráficos acusan una tendencia superior entre los L1 de ciudad frente a los del resto de Mallorca. Este hecho se debería poder explicar por las ocasiones de consumo cultural que ofrece la ciudad, superior al resto de Mallorca.

\subsection{Hábitos del Grupo encuestado, según lugar de residencia}

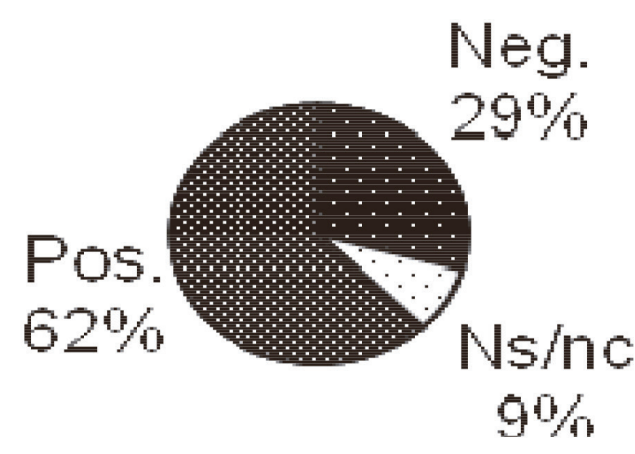

Figura 6. L1 (ciudad)

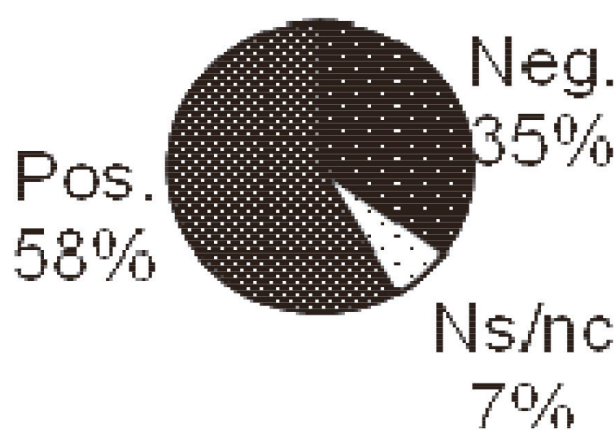

Figura 7. L1 (resto de Mallorca)

\footnotetext{
21 Según las tesis de Flors Mas y Vila i Moreno (2014, 189), la autoconfianza en el uso de una lengua suele ser el principal motivo que aducen los adolescentes de L1 castellana.
} 


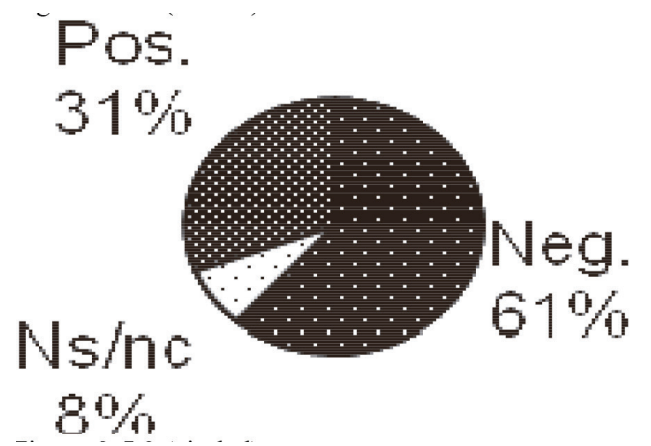

Figura 8. L2 (ciudad)

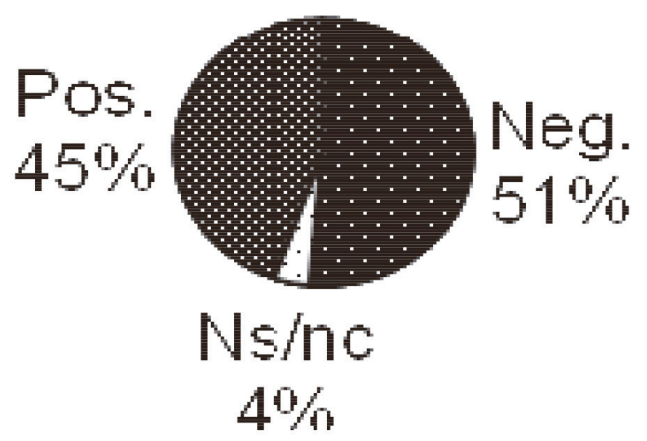

Figura 9. L2 (resto de Mallorca)

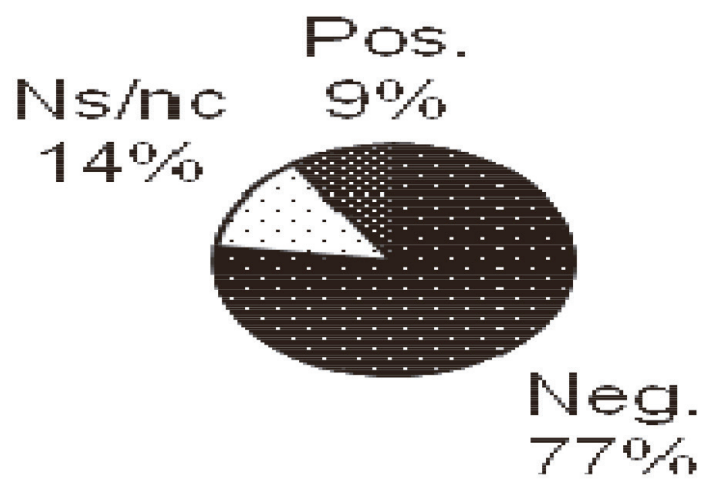

Figura 10. L3 (ciudad)

Los resultados de la Enquesta d'actituds, establecen que los L1 ven más películas y series en versión original catalana. Así, el $50 \%$ de los residentes en Palma y el $17 \%$ de los habitantes del resto de la isla se inclinan por esta opción. La diferencia entre ambos colectivos es que los del resto de Mallorca que manifiestan hacerlo "a veces" son el $67 \%$, mientras que este porcentaje se reduce al $33 \%$ entre los palmesanos. Ningún encuestado del grupo L2 ha indicado que lo haga "normalmente". Se han decantado por la opción "a veces" en un $38 \%$ los habitantes de la ciudad y en un $33 \%$ los del resto de la isla. Los del colectivo L3 no han manifestado hacerlo ni "normalmente" ni "a veces".

Si expresamos los datos en su totalidad, la Enquesta d'actituds revela un consumo mayor en castellano. No se debe olvidar que la ley de mercado, generalmente, se rige por pautas lingüísticas monolingües en lengua estatal: los encuestados de L1 -50 \% "normalmente" y $33 \%$ "siempre" para la ciudad, y $67 \%$ "normalmente" para el resto de la isla-y L2 -31\% "normalmente" y $54 \%$ "siempre" para Palma, y $100 \%$ "siempre" para el resto de Mallorca- ven películas en español como lengua original.

Cabe hacer hincapié, según se desprende de las preguntas 52 y 53, que los L1 consumen más productos culturales en terceras lenguas, mostrando una disposición al plurilingüismo. Cuando residen en la ciudad, estos ven películas y series en ver- 
sión original en inglés subtituladas en catalán en un $34 \%$ y en español en un $50 \%$, porcentajes que se reducen al $0 \%$ y al $17 \%$ respectivamente cuando viven en el resto de Mallorca. Los porcentajes correspondientes para los L2 son del $0 \%$ subtituladas en catalán, y del $23 \%$, en castellano, cuando habitan en el área urbana; y del $0 \%$ y del $33 \%$, respectivamente, en las zonas más rurales. Tan solo un $12.5 \%$ de los L3 consumiría productos audiovisuales en versión original de manera habitual, y subtitulada en español.

Por lo que a la música se refiere, “¿Escuchas música en catalán?”, el consumo se muestra proporcional al contacto con esta lengua, sobre todo entre los L2. El $67 \%$ de los L1 de ciudad declaran escuchar regularmente música en catalán, frente a un $33 \%$ de los del resto de Mallorca. Estos últimos afirman, en un $50 \%$, consumirla "normalmente". Cuando el catalán es una L2 entre los que residen en la ciudad, el $8 \%$ responde escucharla regularmente; si viven en el resto de la isla, el porcentaje aumenta al $67 \%$. Solo el $12.5 \%$ de los que tienen el catalán como L3, que equivale a un único sujeto, confiesan escuchar música en catalán, "normalmente", porcentaje que se corresponde con los que consumen productos audiovisuales en versión original. En lo referente a los hábitos lingüísticos de los L2, seguimos detectando el contraste entre la ciudad y el resto de la isla. Este colectivo muestra una disposición hacía el catalán en un entorno controlado por la socialización primaria.

Finalmente, entre los que tienen el catalán como L1 y como L2, el consumo de música en español o inglés es muy elevado. No hay diferencias significativas entre los de ciudad o los del resto de Mallorca. El grupo de los L3 se inclina por la música en español: el $62.5 \%$ lo hacen "normalmente", y un $15 \%$, "siempre". El consumo en terceras lenguas para estos últimos se distribuye entre un $50 \%$ que lo hace "normalmente", y un $25 \%$, "siempre".

\section{Conclusiones}

A partir del estudio del perfil sociolingüístico de los sujetos de la muestra, se concluye que los L1 de catalán demuestran una predisposición positiva hacia la vehiculación de su lengua. Además, si están sujetos a un entorno controlado por las fuerzas de socialización primaria, clasificado en este estudio como resto de Mallorca, tienden a mostrar cifras altamente positivas hacia la plenitud funcional del catalán. Este tipo de socialización afecta en el mismo sentido a los L2, con un $75 \%$ de las actitudes y un $45 \%$ de los hábitos positivos.

Las elecciones lingüísticas de los sujetos analizados, universitarios de primer año, demuestran que los individuos bilingües, catalán/español, tienden al plurilingüismo, haciendo uso del inglés como lengua de comunicación internacional. El colectivo L3, por las características sociolingüísticas descritas, ámbito urbano suburbial, muestra una tendencia marcada hacia el monolingüismo.

En cuanto a los hábitos de consumo cultural, los resultados para el catalán son inferiores en todos los grupos, llegando a su nulidad entre los L3. La dificultad de acceder a productos audiovisuales en catalán - esta función recae, casi de manera exclusiva, sobre las televisiones autonómicas públicas-, explicaría el desnivel que muestran las cifras, así como el resultado inferior entre los del resto de Mallorca. A ello se debe añadir la presión que ejercen las dinámicas de mercado cuya expresión natural se manifiesta en las lenguas de comunicación, español, inglés. 
En las interacciones interpersonales, se observa la norma según la cual un sujeto responde en la misma lengua en la que se le aborda. Aunque, en algunos casos, en entornos rurales principalmente, este hábito resulte positivo para el catalán, la norma tiende a beneficiar al español, sobre todo en ámbitos administrativos y en el trato con desconocidos. Se constata así la práctica del bilingüismo unidireccional o asimétrico.

Según los datos que se revelan en la tabla 2, las actitudes hacia el catalán como lengua funcional y los hábitos lingüísticos de los encuestados son proporcionales al logro de los objetivos marcados en la guía docente de la asignatura, la cual responde al currículo escolar. Estos resultados corroboran que los colectivos que más repiten la asignatura son los que residen en las áreas más urbanas y castellanizadas.

Para asegurar la asunción plena de las competencias requeridas en la asignatura Lengua Catalana y su Didáctica del Grado en Educación Infantil, equivalente a lo dispuesto en el nivel C1 del MECR, se deberían trabajar a nivel curricular y en mayor medida las actitudes lingüísticas y los hábitos del alumnado hacia el catalán, en particular, y hacia la diversidad lingüística, en general.

\section{Bibliografía}

Argenter, Joan A. (ed.) (2014): Enclavaments lingüístics i comunitats locals. El català a Sardenya, al Carxe i entre els gitanos catalans de França, Barcelona, Institut d'Estudis Catalans-Càtedra UNESCO de Llengües i Educació.

Ballester Cardell, María (2016): "Una nova empenta per a l'ús social de la llengua catalana a les Illes Balears. L'avanç inestable, i no sempre lineal, de la normalització lingüística”, en Revista de Llengua i Dret, Journal of Language and Law, 66, 85-104. Disponible en http://revistes.eapc.gencat.cat/index.php/rld/article/view/10.2436-rld.i66.2016.2861/ n66-ballester-ca.pdf [Consulta: 26 de noviembre de 2018].

Bastardas i Boada, Albert (2002): "Política lingüística mundial a l'era de la globalització: diversitat i intercomunicació des de la perspectiva de la "complexitat"”, en Noves SL, Estiu, 1-14. Disponible en http://www.gencat.cat/llengua/noves/noves/hm02estiu/metodologia/bastardas.pdf [Consulta: 26 de noviembre de 2018].

Bastardas i Boada, Albert (2012): "El català i els joves: propostes de política lingüística del Consell Social de la Llengua Catalana", en Treballs de Sociolingüística Catalana, 22, 7792. Disponible en http://www.raco.cat/index.php/TSC/article/view/265396 [Consulta: 26 de noviembre de 2018].

Bibiloni, Gabriel (2014): "La política lingüística del Govern de José Ramón Bauzà", en Revista de Catalunya, 288, 85-96. Disponible en http://www.revistadecatalunya.cat/upload/ editorial/docPDF-27.pdf [Consulta: 26 de noviembre de 2018].

Bibiloni, Gabriel (2015): "Mallorca", en Divèrsia: Revista de la Càtedra sobre Diversitat Social de la Universitat Pompeu Fabra, 7, 120-132. Disponible en http://www.raco.cat/ index.php/diversia/article/viewFile/301304/390877 [Consulta: 26 de noviembre de 2018].

Bourdieu, Pierre y Jean-Claude Passeron (1977): Reproduction in education, society and culture, Londres, Sage.

Bretxa, Vanessa, Llorenç Comajoan y Natxo Sorolla (2009): "De les pràctiques monolingües familiars a la identificació bilingüe: el cas dels preadolescents de Mataró i la Franja”, en Noves SL. Revista de sociolingüística, Hivern, 1/15, 1-15. Disponible en http://dspace. uvic.cat/handle/10854/3481 [Consulta: 26 de noviembre de 2018]. 
Casas-Deseures, Mariona y Llorenç Comajoan Colomé (2015): “Detecció, correcció i justificació d'errors de normativa en l'alumnat universitari: la formació lingüística dels futurs mestres", en Treballs de Sociolingüística Catalana, 25, 175-197. Disponible en http:// www.raco.cat/index.php/TSC/article/view/306459 [Consulta: 26 de noviembre de 2018].

Cea D’Ancona, María Ángeles (2004): Métodos de encuesta: teoría y práctica, errores y mejora, Madrid, Síntesis.

Cotano, Àngela (2000): Les llengües minoritzades d'Europa, Valencia, $3 i 4$.

Council of Europe (2007a): From Lingiuistic Diversity to Plurilingual Education: Guide for the Development of Language Education Polices in Europe (Main Version), Estrasburgo, Language Policy Division. Disponible en https://rm.coe.int/16802fc1c4 [Consulta: 26 de noviembre de 2018].

Council of Europe (2007b): From Lingiuistic Diversity to Plurilingual Education: Guide for the Development of Language Education Polices in Europe (Executive Version), Estrasburgo, Language Policy Division. Disponible en https://rm.coe.int/16806a892c [Consulta: 26 de noviembre de 2018].

Estors Sastre, Laura (2014): "Les actituds lingüístiques segons l'origen dels aprenents de català com a llengua d'acollida", en Treballs de sociolingüística catalana, 24, 153-171. Disponible en http://www.raco.cat/index.php/TSC/article/view/293772/ 382297 [Consulta: 26 de noviembre de 2018].

Flors Mas, Avel·lí y F. Xavier Vila i Moreno (2014): "Justificant les preferències. Com argumenten les opcions lingüístiques els adolescents catalans", en Treballs de Sociolingüística Catalana, 24, 173-199. Disponible en http://www.raco.cat/index.php/TSC/article/ viewFile/293773/382298 [Consulta: 26 de noviembre de 2018].

Gifreu, Josep (2011): "Language Policies in the Public Media and the Promotion of Catalan in the Privet Media, Arts and Information Tchnologies", en Democratic Policies for Language Revitalisation: the Case of Catalan, Strubell, M. y E. Boix-Fuster (eds.), Londres, Palgrave Macmillan, 182-200.

Gifreu, Josep (2014): El català a l'espai de comunicació. El procés de normalització de la llengua catalana als mèdia (1976-2013), Bellaterra, Publicacions de la Universitat Autònoma de Barcelona, Publicacions de la Universitat Jaume I, Universitat Pompeu Fabra.

Govern de les Illes Balears (1997): “14003 Decret 92/1997, de 4 de juliol, que regula l'ús i l'ensenyament de i en llengua catalana, pròpia de les illes Balears, en els centres docents no universitaris de les illes balears", en Butlletí Oficial de les Illes Balears, 89. Disponible en http://boib.caib.es/pdf/1997089/mp11261.pdf [Consulta: 26 de noviembre de 2018].

Govern de les Illes Balears (2013): “7432 Decret 15/2013, de 19 d'abril, pel qual es regula el tractament integrat de les llengües als centres docents no universitaris de les Illes Balears", en Butlletí Oficial de les Illes Balears, 053. Disponible en http://www.caib.es/ eboibfront/ca/2013/8117/514453/decret-15-2013-de-19-d-abril-pel-qual-es-regula-el [Consulta: 26 de noviembre de 2018].

Ibáñez Ferreté, Òscar-Adrià (2014): “L'estudi d'ideologies lingüístiques amb grups de discussió: el cas dels estudiants palmesans de ciències socials de la Universitat de les Illes Balears", en Treballs de Sociolingüística Catalana, 24, 225-240. Disponible en http:// www.raco.cat/index.php/TSC/article/view/293775 [Consulta: 26 de noviembre de 2018].

Iranzo García, Pilar y Enric Queralt i Catà (2010): “Competència comunicativa lingüística. Ensenyar i aprendre a ser competent lingüísticament a l'educació obligatòria", en Revista Catalana de Pedagogia, 7, 55-79. Disponible en http://www.raco.cat/index.php/RevistaPedagogia/article/view/252221 [Consulta: 26 de noviembre de 2018]. 
Joan i Marí, Bernat (2008): “Acció cívica per fomentar actituds lingüístiques positives”, en Revista Catalana de Pedagogia, 6, 25-36. Disponible en http:/www.raco.cat/index.php/ RevistaPedagogia/article/view/212430 [Consulta: 26 de noviembre de 2018].

Marimon Riutort, Antoni (2015): "Dels murcians als andalusos. La immigració peninsular a les illes Balears (1930-1980)", en Cercles: Revista d'Història Cultural, 18, 79-99. Disponible en http://www.raco.cat/index.php/Cercles/article/view/298913 [Consulta: 26 de noviembre de 2018].

Melià, Joan (2001): "La política lingüística del govern de les Illes Balears”, en Llengua i Ús: Revista Tècnica de Política Lingüística, 20, 4-11. Disponible en http://www.raco.cat/index.php/LlenguaUs/article/view/127851/177215 [Consulta: 26 de noviembre de 2018].

Melià, Joan (2015): "El català a les Illes Balears. 2014, primera aproximació", en Llengua i Ús: Revista Tècnica de Política Lingüística, 57, 53-74. Disponible en http://www.raco. cat/index.php/LlenguaUs/article/view/300745 [Consulta: 26 de noviembre de 2018].

Moreno Cabrera, Juan Carlos (2009): "Bilingüismo e lingua común”, en Lingua e docencia universitaria: V Xornadas sobre Lingua e Usos: A Coruña, 12-14 de novembro de 2008, X. M. Mosquera Carregal, X. M. y S. Pino Ramos (coords.), 163-177. Disponible en http://ruc.udc.es/dspace/bitstream/handle/2183/8855/CC103art16.pdf?sequence=1 [Consulta: 26 de noviembre de 2018].

Munar i Munar, Felip (2009): “Dades sobre l'ensenyament infantil i primari a les Illes Balears", en Treballs de Sociolingüística Catalana, 20, 71-96. Disponible en http://www. raco.cat/index.php/TSC/article/view/229935 [Consulta: 26 de noviembre de 2018].

Navarro, José Luis, Ángel Huguet y Clara Sansó (2016): "Procesos de interdependencia entre lenguas. El caso del alumnado inmigrante en Cataluña", en Revista de Psicodidáctica, 21/2, 227-243. Disponible en http://www.ehu.eus/ojs/index.php/psicodidactica/article/ view/13396 [Consulta: 26 de noviembre de 2018].

Parcerisa, Lluís y Antoni Verger Planells (2016): “"Contra la imposició, defensem l’educació”: Crònica de la mobilització educativa a les Illes Balears (2013-2015)", en Anuari de l'Educació de les Illes Balears, 2016, 354-373. Disponible en https:/dialnet.unirioja.es/ servlet/articulo?codigo=5794884 [Consulta: 26 de noviembre de 2018].

Poggeschi, Giovanni (2004): "La política lingüística a Letònia”, en Noves SL. Revista de Sociolingüística, Tardor, 1-9. Disponible en http://www.gencat.cat/llengua/noves/noves/ hm04tardor/docs/c_poggeschi.pdf [Consulta: 26 de noviembre de 2018].

Pradilla Cardona, Miquel Àngel (2017): "El model lingüístic educatiu a Catalunya el segle xxi”, en Revista de Filología Valenciana, 1, 223-239. Disponible en http://revistavalencianadefilologia.net/index.php/rvf/article/view/28 [Consulta: 26 de noviembre de 2018]. 


\section{Anexo}

\section{Cuestionario anónimo}

* Municipio de residencia (además, si el municipio es Palma, Calvià, Llucmajor o Marratxí, indica también el barrio).

\section{Marca solo una opción como respuesta}

1. Edad. (A: 18 o meNOS; B: 19-20; C: 21-22; D: 23-24; E: 25 o más.)

2. Comunidad autónoma o país de nacimiento. (A: Illes Balears; B: otra comunidad autónoma de habla catalana; C: otra comunidad autónoma; D: otro país de habla hispana; E: otro país.)

3. Vía de acceso a la Universidad. (A: bachillerato; B: formación profesional; C: mayores de 25 años; D: otra.)

4. ¿Es la primera vez que cursas esta asignatura? (A: sí; B: no.)

\section{(A: muy mal; B: mal; C: regular; D: bien; E: muy bien.)}

5. ¿Cómo hablas el catalán?

6. ¿Cómo entiendes el catalán?

7. ¿Cómo escribes en catalán?

8. ¿Cómo lees en catalán?

9. ¿Cómo hablas el español?

10. ¿Cómo entiendes el español?

11.¿Cómo escribes en español?

12.¿Cómo lees en español?

\section{(A: catalán; B: español; C: inglés; D: alemán; E: otra lengua.)}

13. Cuando te diriges a una persona de los siguientes grupos sociales por primera vez y sin conocerla, ¿qué lengua utilizas?

14. Turistas.

15. Vecinos.

16.Compañeros de trabajo.

17. Gente que no conozco por la calle.

18.Personal sanitario.

19. Trabajadores públicos.

20. Alguien que llama a la puerta.

21.Policías.

22.Profesores.

23.Personal de la Universidad.

24. Vendedores.

25.Cuando una persona de los siguientes grupos sociales se te dirige en catalán, ¿en qué lengua le respondes?

26. Turistas.

27. Vecinos.

28. Compañeros de trabajo. 
29. Gente que no conozco por la calle.

30.Personal sanitario.

31. Trabajadores públicos.

32. Alguien que llama a la puerta.

33.Policías.

34.Profesores.

35.Personal de la Universidad.

36. Vendedores.

37. Cuando una persona de los siguientes grupos sociales se te dirige en español, ¿en qué lengua le respondes?

38. Turistas.

39. Vecinos.

40.Compañeros de trabajo.

41. Gente que no conozco por la calle.

42.Personal sanitario.

43. Trabajadores públicos.

44. Alguien que llama a la puerta.

45.Policías.

46.Profesores.

47.Personal de la Universidad.

48. Vendedores.

\section{(A: nunca; B: raramente; C: a veces; D: normalmente; E: siempre.)}

49.¿Hablas catalán en casa?

50.¿Ves películas o series en catalán como versión original?

51.¿Ves películas o series en español como versión original?

52.¿Ves películas o series en una tercera lengua subtituladas en catalán?

53. ¿Ves películas o series en una tercera lengua subtituladas en español?

54. ¿Ves películas o series dobladas en catalán?

55.¿Ves películas o series dobladas en español?

56. ¿Ves otros programas de televisión en catalán diferentes a las películas o series?

57. ¿Ves otros programas de televisión en español diferentes a las películas o series?

58.Las películas que ves en catalán, ¿suelen ser en la televisión?

59.Las películas que ves en catalán, ¿suelen ser en el cine?

60. ¿Escuchas música en catalán?

61.¿Escuchas música en español?

62. ¿Escuchas música en una tercera lengua?

(A: totalmente en desacuerdo; B: en desacuerdo; $C$ : ni de acuerdo ni en desacuerdo; D: de acuerdo; E: totalmente de acuerdo.)

63.Me gusta escuchar el catalán alrededor mío, aunque a veces no lo entienda todo.

64.Me gusta escuchar música en catalán, aunque a veces no lo entienda todo.

65.Me gusta ver películas y series en catalán, aunque a veces no lo entienda todo. 66. Me gusta que se me dirijan en catalán desde el primer momento. 
67. Creo que es normal que el catalán se pida en el sector público.

68. Creo que es normal que el español se pida en el sector público.

69. Creo que es normal que el catalán se pida en el sector privado.

70. Creo que es normal que el español se pida en el sector privado.

71. Creo que es normal que una asignatura se imparta en catalán, aunque parte de la clase no lo tenga como primera lengua.

72. Creo que todo tendría que estar rotulado en catalán, como mínimo, sin perjuicio de que lo esté en otras lenguas también.

73. Creo que todo tendría que estar rotulado en español, como mínimo, sin perjuicio de que lo esté en otras lenguas también. 